\title{
Turoctocog alfa: an evidence-based review of its potential in the treatment of hemophilia A
}

This article was published in the following Dove Press journal:

Drug Design, Development and Therapy

24 March 2015

Number of times this article has been viewed

\section{Hideyuki Takedani Jun Hirose}

Department of Joint Surgery, Research Hospital of the Institute of Medical Science, The University of Tokyo, Tokyo, Japan
Correspondence: Hideyuki Takedani Department of Joint Surgery, Research Hospital of the Institute of Medical Science, The University of Tokyo, 4-6-I Shirokanedai Minato-ku, Tokyo,

108-8639, Japan

Tel +8I 334438 I II

Fax +81364092402

Email takedani@ims.u-tokyo.ac.jp

\begin{abstract}
Turoctocog alfa is the first B-domain-truncated third generation recombinant coagulation factor VIII (FVIII) product. Nonclinical in vitro and animal model studies have demonstrated that turoctocog alfa has similar functional potency and hemostatic efficacy as comparator FVIII products. With respect to discrepancies in the level of FVIII concentrate in plasma of current FVIII products on comparing measurement results between one-stage clot and chromogenic assays, there was no difference in the in vitro turoctocog alfa study; however, measured FVIII concentrate in field study was higher with the chromogenic assay $(1.08 \mathrm{IU} / \mathrm{mL})$ than with one-stage assay $(0.83 \mathrm{IU} / \mathrm{mL})$. Two published clinical studies on previously treated patients (PTPs) and clinical pharmacokinetics have described that the pharmacokinetic parameters are similar, and the safety and efficacy for prevention and treatment for bleeding are also similar to those of standard half-life FVIII products. Three clinical trials are ongoing to assess the long-term safety and efficacy of turoctocog alfa for PTPs and previously untreated patients. Those data will be published in the near future, and it will be possible to use turoctocog alfa for all hemophilia patients. However, studies will be needed to confirm the turoctocog alfa profile, such as the stability of dissolved turoctocog alfa over 24 hours at room temperature and post-marketing clinical research aimed at meeting Europe Medicines Agency post-marketing safety and efficacy requirements in PTPs. It is recommended to wait before using turoctocog alfa for previously untreated patients and major surgery until further data have been collected and published.
\end{abstract}

Keywords: hemophilia A, B-domain-truncated, third generation factor VIII product, previous untreated patients, chromogenic assay

\section{Introduction}

Hemophilia $\mathrm{A}$ is a rare $\mathrm{X}$-linked congenital coagulation disease caused by complete or partial deficiency of coagulation factor VIII (FVIII). ${ }^{1,2}$ According to the plasma FVIII level, hemophilia is classified as severe $(<1 \%)$, moderate $(1 \%-5 \%)$, and mild $(>5 \%) .^{2-4}$ The characteristic symptom of hemophilia is spontaneous bleeding, and hemostatic control is impossible in severe cases without FVIII products. As FVIII products, plasma-derived and recombinant FVIII (rFVIII) products are available, and extended half-life products are undergoing clinical trials. Plasma-derived and some rFVIII products contain animal and/or human components, which have essentially zero risk of transmitting viruses and other blood-borne pathogens, including future potential threats from pathogens yet to be identified. ${ }^{3,5}$

There are two types of FVIII replacement therapy for hemostatic control. They are on-demand therapy (administered on demand after bleeding episodes) and prophylactic therapy (administered prophylactically to prevent bleeds). Prophylactic therapy is recommended as the first-line hemophilia therapy, particularly for hemophilic children. The aim is prevention of serious life threatening and joint damage from bleeding. 
Prophylactic therapy with the first infusion initiated at 2 years or before the second joint bleeding is considered the best preventative treatment for hemophilic joint damage., ${ }^{2,6}$

In the last decade, the manufacturing process for coagulation factor products has improved, and recombinant coagulation factors can be produced with chemically synthesized or recombinant alternated culture media, which contains substitutes for animal or human components (third-generation products). ${ }^{7}$ Furthermore, the B-domain truncation can be easier to purify and characterize than full length molecules. ${ }^{8,9}$ Recently an extended half-life product has been developed. In developed nations, animal or human component-free and highly pure FVIII products are preferred for use, particularly for hemophilic children, as prophylactic therapy.

Turoctocog alfa is the first B-domain-truncated thirdgeneration rFVIII product in the world. Two major clinical trials have been performed and published, ${ }^{10,11}$ and some clinical trials are still ongoing or in preparation. This article has three parts: review of basic characterizations, summary of clinical trials, and a clinical hemostatic control plan based on clinical trial data.

\section{Molecular structural and functional characterization (preclinical evaluation) Molecular structural characterization}

Turoctocog alfa is a B-domain-truncated rFVIII product manufactured without the use of animal or human proteins. The molecule consists of 740 amino acids of the heavy chain containing the A1-A2 domains, 21 amino acids of the truncated $\mathrm{B}$ domain, and 684 amino acids of the light chain containing the $\mathrm{A} 3-\mathrm{C} 1-\mathrm{C} 2$ domains. The 21 amino acid residues of the truncated $\mathrm{B}$ domain are ten amino acid residues from the $\mathrm{N}$ terminus of the $\mathrm{B}$ domain linked to eleven amino acid residues from the $\mathrm{C}$ terminus of the $\mathrm{B}$ domain. ${ }^{7,12}$ The natural full-length $B$ domain consists of 908 amino acids and appears to not be required for effective coagulation; B-domaindeleted FVIII molecules have biological activity. ${ }^{13,14}$ The truncated $\mathrm{B}$ domain is removed by thrombin activation, and turoctocog alfa, an active rFVIII with a structure similar to that of endogenous FVIII, is obtained. ${ }^{7}$

\section{Functional characterization}

Some nonclinical in vitro and animal model studies have demonstrated that turoctocog alfa has similar functional potency and hemostatic efficacy as comparator FVIII products. Based on an in vitro analysis of seven individual batches, the specific FVIII concentrate (FVIII:C) of turoctocog alfa was
Table I Summary of chromogenic/one-stage ratio

\begin{tabular}{|c|c|c|}
\hline & Product & Chromogenic/one-stage ratio \\
\hline \multirow[t]{2}{*}{ Field study ${ }^{18}$} & Advate & 1.19 \\
\hline & Turoctocog alfa & 1.30 \\
\hline \multirow[t]{2}{*}{ PK study ${ }^{7}$} & Advate & 1.0 \\
\hline & Turoctocog alfa & 1.00 \\
\hline
\end{tabular}

Abbreviation: PK, pharmacokinetic.

$9,300 \pm 400 \mathrm{IU} / \mathrm{mg}$ by one-stage clot assay. More analysis showed that the kinetic parameters of turoctocog alfa are similar to those of both a commercially available full-length rFVIII product and a plasma-derived FVIII product. ${ }^{7}$ In a few animal model studies, the pharmacokinetic (PK) profile, hemostatic efficacy, and safety of turoctocog alfa have been evaluated, and it is similar to those of a commercially available full-length rFVIII. ${ }^{15-17}$

Discrepancies in the levels of FVIII:C in plasma of current rFVIII products have been found on comparing measurement results between one-stage clot and chromogenic assays. ${ }^{18}$ An international multicenter randomized comparative field study was conducted to assess the activity and assay variability of turoctocog alfa and a commercially available full-length rFVIII product. ${ }^{10}$ The results showed that levels of FVIII:C in plasma of turoctocog alfa and a commercially available fulllength rFVIII were similar at both low and high concentrations of FVIII:C. Discrepancies in the levels of FVIII:C between turoctocog alfa and other FVIII products have not been found in compared measurement results by one-stage clot assay or chromogenic assay in a similar in vitro study (Table 1$){ }^{7}$

\section{Clinical trial evaluation PK study}

This study was an open-label, first human dose, PK, singledose trial for patients with severe hemophilia A (Table 2). ${ }^{5}$ Patients were 12-55 years and enrolled in four countries. All patients had a history of at least 150 exposure days to FVIII products (previously treated patient [PTP]), without present or past history of inhibitors of FVIII (non-inhibitor: $<0.6$ Bethesda unit $(\mathrm{BU}) / \mathrm{mL})$. The primary endpoints of this PK study were incremental recovery, terminal half-life, area under the plasma concentration-time curve (hour $[\mathrm{h}] \times \mathrm{IU} / \mathrm{mL}$ ), and clearance $(\mathrm{mL} / \mathrm{h})$. The secondary endpoints were maximum concentration $(\mathrm{IU} / \mathrm{mL})$, mean residence time $(\mathrm{h})$, and volume of distribution at steady state (mL). FVIII:C was measured in a one-stage assay. These parameters were compared with those of another recombinant third-generation product but not a B-domain-deleted FVIII product. All of them were similar between the two third-generation products. 
Table 2 Summary of pharmacokinetic study

\begin{tabular}{|c|c|c|}
\hline Pharmacokinetic data & $\begin{array}{l}\text { Turoctocog alfa } \\
\text { Mean (SD) }\end{array}$ & $\begin{array}{l}\text { Advate } \\
\text { Mean (SD) }\end{array}$ \\
\hline $\begin{array}{l}\text { Incremental recovery } \\
(\mathrm{IU} / \mathrm{mL}) /(\mathrm{IU} / \mathrm{mL})\end{array}$ & $0.019(0.002)$ & $0.019(0.003)$ \\
\hline $\mathrm{AUC}(\mathrm{h} / \mathrm{IU} / \mathrm{mL})$ & $12.97(3.48)$ & $13.03(4.25)$ \\
\hline Half-life (h) & $10.83(4.95)$ & $11.19(3.5 I)$ \\
\hline $\mathrm{CL}(\mathrm{mL} / \mathrm{h})$ & $302.3(98.12)$ & $307.0(100.2)$ \\
\hline $\mathrm{C}_{\max }(\mathrm{IU} / \mathrm{mL})$ & $0.99(0.15)$ & $1.02(0.13)$ \\
\hline MRT (h) & |5.7| (6.38) & I5.79 (4.74) \\
\hline Vss (mL) & $4,284(748.6)$ & $4,466(801.7)$ \\
\hline
\end{tabular}

\section{Guardian $^{\mathrm{TM}}$ I}

This clinical trial was a multicenter, multinational, open-label, non-controlled trial for patients with severe hemophilia A. ${ }^{19}$ The 150 participating patients were aged $12-65$ years and enrolled in 15 countries. All patients were PTPs and noninhibitors. Patients with a history of diseases, increased risk of thromboembolic events, or risk of opportunistic disease (CD4 cell counts $\leq 200 / \mu \mathrm{L}$ ) were excluded. All patients received prophylaxis with turoctocog alfa $(20-40 \mathrm{IU} / \mathrm{kg}$ every second day or 20-50 IU/kg three times weekly) for approximately 6 months. This study was conducted to assess the safety and efficacy of turoctocog alfa for prevention and control of bleeding.

With respect to safety, nine serious adverse events of a total of 225 adverse events were reported in seven patients. Of these, four events in two patients were evaluated by the investigator as probably associated with turoctocog alfa. One patient showed hypertension, sinus tachycardia, and insomnia and another showed an increase of hepatic enzyme. No patients developed FVIII inhibitors during the trial, and no safety concern was raised by physical examinations or clinical laboratory tests.

With respect to hemostatic efficacy of bleeding episodes (Table 3), 499 bleeding episodes were recorded in 105 (70\%) patients, the median annualized bleeding rate was 3.7 bleeds/ patient/year, and the estimated mean annualized bleeding rate was 6.5 bleeds/patient/year. These bleeding rates are significantly lower than those reported for patients previously treated by on-demand treatment. ${ }^{20-22}$ Of the patients, $30 \%$ (45) did not experience a bleeding episode during the trial. The most frequent location of a bleeding episode was in joints (78\%) and the vast majority $(90 \%)$ of the episodes was classified as mild or moderate. Of the episodes, 446 (86\%) were resolved with 1-2 infusions of turoctocog alfa.
Table 3 Summary of bleeding episodes and hemostatic response to turoctocog alfa treatment

\begin{tabular}{lll}
\hline & Number & $\%$ \\
\hline Patients & 150 & 100 \\
Bleeding episodes & 499 & 100 \\
Major cause of bleeds & & \\
$\quad$ Spontaneous & 332 & 66.5 \\
$\quad$ Traumatic & 124 & 24.8 \\
Joint bleeds & 389 & 78.0 \\
$\quad$ Target joint & 325 & 65.1 \\
Classification of bleeds & & \\
Severe & 46 & 9.2 \\
$\quad$ Moderate & 449 & 90.0 \\
Hemostatic response & & \\
$\quad$ Excellent & 140 & 28.1 \\
$\quad$ Good & 263 & 52.7 \\
Moderate & 62 & 12.4 \\
None & 12 & 2.4 \\
\hline
\end{tabular}

Note: Data from Lentz et al..$^{19}$

For 403 (81\%) of the episodes, treatment was considered a success (excellent or good response). The mean consumption of turoctocog alfa until bleeding cessation was $45.6 \mathrm{IU} / \mathrm{kg} /$ episode. The mean consumption per patient of turoctocog alfa for prophylactic treatment was $309 \mathrm{IU} / \mathrm{kg} /$ month, with a mean single dose of $24.4 \mathrm{IU} / \mathrm{kg}$.

This study incorporated a sub-trial study in which the safety and efficacy of prevention and treatment of bleeding during surgical procedures were assessed. The result is described in the section "Surgical trials".

\section{Guardian $^{\text {TM }} 2$}

This study is a multicenter, multinational, open-label, long-term safety, efficacy study for participating patients in Guardian ${ }^{\mathrm{TM}} 1$ and 3. Thus, this Guardian ${ }^{\mathrm{TM}} 2$ (ongoing trial, unpublished data) is an extension trial of Guardian ${ }^{\mathrm{TM}}$ 1 and 3.

\section{Guardian ${ }^{\mathrm{TM}} 3$}

This study was a multicenter, multinational, uncontrolled, open-label trial for patients with severe hemophilia A. ${ }^{11}$ All patients were aged less than 11 years and in two age cohorts: younger children ( $0-5$ years) and older children (6-11 years). All 60 patients were PTPs and non-inhibitors. The younger cohort had 29 children and the older had 31 . All patients received turoctocog alfa prophylaxis (25-50 $\mathrm{IU} / \mathrm{kg}$ every second day or 25-60 IU $/ \mathrm{kg}$ three times weekly). This study was conducted to assess the safety and efficacy of turoctocog alfa for prevention and control of bleeding and evaluate PK parameters in children.

With respect to safety, a total of 86 adverse events were reported by 32 patients. Of them, two possible related 
non-serious adverse events (incorrect dose administration and a contusion) occurred in one patient. Three unlikely related serious adverse events (soft tissue injury, viral gastroenteritis, and device-related infection) occurred in three patients who recovered. During the trial, one patient had an unconfirmed positive FVIII inhibitor test $(1.3 \mathrm{BU} / \mathrm{mL})$, but the next test 11 days later was negative. Thus, no patient developed inhibitors during the trial. No FVIII inhibitors were detected and the one-side $97.5 \%$ upper confidence limit for the inhibitor incidence rate of zero was $6.1 \%$.

With respect to hemostatic efficacy of bleeding episodes, 126 episodes were recorded in 41 (65\%) patients of which 53 episodes were in younger and 73 in older children. The mean annualized bleeding rate was 3.0 and the estimated mean annualized bleeding rate 5.3 bleeds/patient/year. The mean bleeding rate of spontaneous bleeds appeared lower in the younger cohort than in the older cohort. Of the patients, 22 (35\%) (eleven younger and eleven older children) did not experience a bleeding episode during the trial. The most frequent location of bleeding episode was in joints ( $45.3 \%$ of younger cohort and $47.9 \%$ of older cohort), and the vast majority of bleeding episodes were classified as mild or moderate (more than $90 \%$ of both cohorts). Of the bleeding episodes, 120 (95\%) were resolved by 1-2 infusions of turoctocog alfa. For 116 (92\%) episodes, treatments were considered as successful. The mean consumption of turoctocog alfa until bleeding cessation was $54.2 \mathrm{IU} / \mathrm{kg} / \mathrm{episode}$. The mean consumption per patient of turoctocog alfa for prophylactic treatment was $462 \mathrm{IU} / \mathrm{kg} / \mathrm{month}$, with a mean single dose of $36.8 \mathrm{IU} / \mathrm{kg}$.

With respect to PK parameters, these parameters of turoctocog alfa were comparable between the younger and older cohorts and were similar to those of previous FVIII products. However, measured FVIII:C in a PK test was generally higher by the chromogenic assay than with the one-stage assay.

This study incorporated a sub-trial study in which the safety and efficacy of prevention and treatment of bleeding during surgical procedures were assessed. The result is described in the "Surgical trials" section.

\section{Surgical trials}

Forty-one surgical procedures were performed in 33 patients aged 4-59 years. ${ }^{23}$ Of the 41 procedures, 15 were major surgeries in 13 patients, and 26 were minor surgeries in 21 patients. Most of the major surgeries were five total joint arthroplasties. Most minor surgeries were 19 dental treatments. In the perioperative period, turoctocog alfa was administered by bolus infusion or continuous infusion.
Dosing was determined by the investigator based on local practice. Hemostatic control in the perioperative periods was considered successful (with excellent or good response) for all of both major and minor surgeries. No safety issues were identified. No patients developed FVIII inhibitors or thrombosis in the perioperative period. Five mild or moderate adverse events were recorded in five patients during major surgery and evaluated as unlikely to have been associated with turoctocog alfa.

\section{Ongoing trials}

There are three recruiting and ongoing studies (Guardian ${ }^{\text {TM }}$ 2, 4 and 5). Guardian ${ }^{\mathrm{TM}} 4$ is intended to assess the safety and efficacy of turoctocog alfa for previously untreated patients (PUPs). Guardian ${ }^{\mathrm{TM}} 5$ is assessing the safety and efficacy of turoctocog alfa in the long-term prevention and treatment of bleeding episodes.

\section{Clinical hemostatic control plan based on clinical trial data Daily hemostatic control by on-demand and prophylactic therapy}

According to the PK study and Guardian ${ }^{\mathrm{TM}} 1$ and 3 trials, PK parameters of turoctocog alfa are similar to those of previous FVIII products. The efficacy of turoctocog alfa for PTPs is similar to that of previous FVIII products not only for prevention and treatment of bleeding episodes but also for health-related quality of life of patients using HaemophiliaQuality of Life (HAEMO-QOL). ${ }^{24}$ Most reported adverse events in clinical trials for PTPs were mild or moderate and unlikely to be associated with turoctocog alfa when it was administered for daily prevention and treatment of bleeding. Development of inhibitors and thrombosis has also not occurred during clinical trials. The administration dose and interval of turoctocog alfa are the same as those of previous FVIII products. Turoctocog alfa has a unique larger content specification. Its size is $3,000 \mathrm{IU} / 4 \mathrm{~mL}$ and its concentration is highest among all FVIII products. This characteristic is better for heavy patients, owing to the smaller injection volume and fewer dissolution steps.

Turoctocog alfa will thus be a preferred product for PTPs, particularly heavy patients. For PUPs a clinical trial is ongoing and recruiting (Guardian ${ }^{\mathrm{TM}} 4$ ), therefore, it is better to delay use of turoctocog alfa until the result is published.

\section{Surgery}

Usually two administration methods during surgery are available: bolus and continuous. Theoretically and clinically, 
continuous infusion is better than bolus infusion because FVIII:C remains at its target level without excessively high peaks or risky low troughs..$^{25,26}$ Planning of both bolus and continuous administration of turoctocog alfa during the perioperative period will be easy because the PK parameters of turoctocog alfa are similar to those of previous FVIII products and will be sufficient for controlling bleeding, according to the bolus administration plan. However, there is a risk in controlling bleeding according to a continuous administration plan because there is no stability data for dissolved turoctocog alfa over 24 hours at room temperature. The surfactant contained in FVIII products prevents settling of FVIII as a high molecular weight protein. This tendency is more severe for turoctocog alfa than for previous FVIII products, owing to the higher concentration of turoctocog alfa. Another concern is the difference in measured FVIII:C between one-stage and chromogenic assays. A preclinical study showed no discrepancies in the level of FVIII:C between turoctocog alfa and other FVIII products in compared measurement results of one-stage clot and chromogenic assays. However, measured FVIII using chromogenic assay was higher than that using one-stage assay in a clinical study in children. ${ }^{11}$ This difference is a major issue because FVIII:C is an important guide value for hemostatic control, particular during surgery. Before the discrepancy issue can be resolved, not only evaluation of FVIII:C but observation of the degree of bleeding from surgical wounds are important for perioperative hemostatic control.

Thus, turoctocog alfa is a product for hemostatic control in surgery using the bolus administration plan, but it may be too soon to administer turoctocog alfa continuously, at least until stability data have been published.

\section{Conclusion}

Turoctocog alfa is the first B-domain-truncated third generation rFVIII product worldwide. Two published clinical studies for PTPs and clinical PK have suggested that the PK parameters and the safety and efficacy for prevention and treatment of bleeding are similar to those of previous FVIII products. Five clinical trials are ongoing or in planning stage to assess the safety and efficacy of turoctocog alfa for PTPs over the long term and for PUPs. Those data will be published in the near future and turoctocog alfa will potentially be a more reliable FVIII product. However, studies will be needed to confirm the turoctocog alfa profile, including its stability in solution over 24 hours at room temperature, in addition to post-marketing clinical research studies. Turoctocog alfa is a preferred product to use for hemophilic PTPs. It is recommended to wait to use turoctocog alfa for PTPs and major surgery until further data have been accumulated and published.

\section{Acknowledgments}

The authors gratefully acknowledge Mitsuhiro Kuwahara for review support of revised article. He is an employee of Novo Nordisk.

\section{Disclosure}

The authors report no conflicts of interest in this work.

\section{References}

1. Mannucci PM, Tuddenham EG. The hemophilias - from royal genes to gene therapy. N Engl J Med. 2001;344(23):1773-1779.

2. Srivastava A, Brewer AK, Mauser-Bunschoten EP, et al. Guidelines for the management of hemophilia. Haemophilia. 2013;19(1):e1-e47.

3. Coppola A, Di Capua M, Di Minno MN, et al. Treatment of hemophilia: a review of current advances and ongoing issues. $J$ Blood Med. 2010;1:183-195.

4. Pipe S. Antihemophilic factor (recombinant) plasma/albumin-free method for the management and prevention of bleeding episodes in patients with hemophilia A. Biologics. 2009;3:117-125.

5. Martinowitz U, Bjerre J, Brand B, et al. Bioequivalence between two serum-free recombinant factor VIII preparations (N8 and ADVATE ${ }^{\mathbb{B}}$ )an open-label, sequential dosing pharmacokinetic study in patients with severe haemophilia A. Haemophilia. 2011;17(6):854-859.

6. Berntorp E, Boulyjenkov V, Brettler D, et al. Modern treatment of haemophilia. Bull World Health Organ. 1995;73(5):691-701.

7. Christiansen ML, Balling KW, Persson E, et al. Functional characteristics of N8, a new recombinant FVIII. Haemophilia. 2010;16(6): 878-887.

8. Fang H, Wang L, Wang $\mathrm{H}$. The protein structure and effect of factor VIII. Thromb Res. 2007;119(1): 1-13.

9. Jankowski MA, Patel H, Rouse JC, Marzilli LA, Weston SB, Sharpe PJ. Defining 'full-length' recombinant factor VIII: a comparative structural analysis. Haemophilia. 2007;13(1):30-37.

10. Viuff D, Barrowcliffe T, Saugstrup T, Ezban M, Lillicrap D. International comparative field study of N8 evaluating factor VIII assay performance. Haemophilia. 2011;17(4):695-702.

11. Kulkarni R, Karim FA, Glamocanin S, et al. Results from a large multinational clinical trial (guardian ${ }^{\mathrm{TM}} 3$ ) using prophylactic treatment with turoctocog alfa in paediatric patients with severe haemophilia A: safety, efficacy and pharmacokinetics. Haemophilia. 2013;19(5):698-705.

12. Thim L, Vandahl B, Karlsson J, et al. Purification and characterization of a new recombinant factor VIII (N8). Haemophilia. 2010;16(2): 349-359.

13. Burke RL, Pachl C, Quiroga M, et al. The functional domains of coagulation factor VIII:C. J Biol Chem. 1986;261(27):12574-12578.

14. Pittman DD, Alderman EM, Tomkinson KN, Wang JH, Giles AR, Kaufman RJ. Biochemical, immunological, and in vivo functional characterization of B-domain-deleted factor VIII. Blood. 1993;81(11):2925-2935.

15. Elm T, Karpf DM, Ovlisen K, et al. Pharmacokinetics and pharmacodynamics of a new recombinant FVIII (N8) in haemophilia A mice. Haemophilia. 2012;18(1):139-345.

16. Karpf DM, Kjalke M, Thim L, et al. Pharmacokinetics and ex vivo whole blood clot formation of a new recombinant FVIII (N8) in haemophilia A dogs. Haemophilia. 2011;17(5):e963-e968.

17. Pastoft AE, Lykkesfeldt J, Ezban M, Tranholm M, Whinna HC, Lauritzen B. A sensitive venous bleeding model in haemophilia A mice: effects of two recombinant FVIII products (N8 and Advate $\left({ }^{\circledR}\right)$ ). Haemophilia. 2012;18(5):782-788. 
18. Ingerslev J, Jankowski MA, Weston SB, Charles LA, ReFacto Field Study Participants. Collaborative field study on the utility of a BDD factor VIII concentrate standard in the estimation of BDDr Factor VIII:C activity in hemophilic plasma using one-stage clotting assays. J Thromb Haemost. 2004;2(4):623-628.

19. Lentz SR, Misgav M, Ozelo M, et al. Results from a large multinational clinical trial (guardian ${ }^{\mathrm{TM}} 1$ ) using prophylactic treatment with turoctocog alfa in adolescent and adult patients with severe haemophilia A: safety and efficacy. Haemophilia. 2013;19(5):691-697.

20. Abshire TC, Brackmann HH, Scharrer I, et al. Sucrose formulated recombinant human antihemophilic factor VIII is safe and efficacious for treatment of hemophilia A in home therapy - International Kogenate-FS Study Group. Thromb Haemost. 2000;83(6):811-816.

21. Lusher JM, Lee CA, Kessler CM, Bedrosian CL, ReFacto Phase 3 Study G. The safety and efficacy of B-domain deleted recombinant factor VIII concentrate in patients with severe haemophilia A. Haemophilia. 2003; $9(1): 38-49$.

22. Valentino LA, Mamonov V, Hellmann A, et al. A randomized comparison of two prophylaxis regimens and a paired comparison of on-demand and prophylaxis treatments in hemophilia A management. $J$ Thromb Haemost. 2012;10(3):359-367.
23. Santagostino E, Lentz SR, Misgav M, et al. Safety and efficacy of turoctocog alfa (NovoEight) during surgery in patients with haemophilia A: results from the multinational guardian clinical trials. Haemophilia. 2015;21(1):34-40.

24. Santagostino E, Lentz SR, Busk AK, Regnault A, Iorio A. Assessment of the impact of treatment on quality of life of patients with haemophilia A at different ages: insights from two clinical trials on turoctocog alfa. Haemophilia. 2014;20(4):527-534.

25. Meijer M, Rauchensteiner S, Santagostino E, et al. Continuous infusion of recombinant factor VIII formulated with sucrose in surgery: non-interventional, observational study in patients with severe haemophilia A. Haemophilia. 2015;21(1):e19-e25.

26. Takedani H. Continuous infusion during total joint arthroplasty in Japanese haemophilia A patients: comparison study among two recombinants and one plasma-derived factor VIII. Haemophilia. 2010;16(5):740-746.
Drug Design, Development and Therapy

\section{Publish your work in this journal}

Drug Design, Development and Therapy is an international, peerreviewed open-access journal that spans the spectrum of drug design and development through to clinical applications. Clinical outcomes, patient safety, and programs for the development and effective, safe, and sustained use of medicines are a feature of the journal, which

\section{Dovepress}

has also been accepted for indexing on PubMed Central. The manuscript management system is completely online and includes a very quick and fair peer-review system, which is all easy to use. Visit http://www.dovepress.com/testimonials.php to read real quotes from published authors.

Submit your manuscript here: http://www.dovepress.com/drug-design-development-and-therapy-journal 